\title{
System-Level Motor Drive Modelling for Optimization-based Designs
}

\author{
Benjamin Cheong, Paolo Giangrande, Xiaochen Zhang, \\ Michael Galea, Pericle Zanchetta, Patrick Wheeler \\ Power Electronics, Machine and Control Group (PEMC), University of Nottingham, \\ University Park Campus, NG7 2RD, Nottingham, United Kingdom \\ Email: Benjamin.cheong@nottingham.ac.uk
}

\section{Acknowledgments}

This project has received funding from the Clean Sky 2 Joint Undertaking under the European Unions Horizon 2020 research and innovation programme under grant agreement no. 807081.

\section{Keywords}

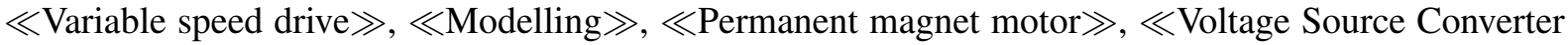
(VSC) $\gg, \ll$ System integration $\gg$

\begin{abstract}
To achieve optimal system weight and efficiency, the design of main components in an AC motor drive (electric machine and power converter) have to be optimized together. This is challenging considering the multiphysics and complex nature of its operation and interactions between the components. There are few papers that address a formal approach towards this design optimization for motor drives at a system-level. This paper aims to fill this gap by presenting a fast and accurate system-level motor drive model, for use in an optimization-based design approach. At a system-level, the presented model is able to consider several aspects of machine-converter interactions. The model is fully analytical, making it very computationally efficient, and its accuracy is validated using a back-to-back motor-drive test setup.
\end{abstract}

\section{Introduction}

Variable speed drives are widely employed in many applications, ranging from home appliance to the More Electric Aircraft (MEA) [1, 2]. A typical state-of-the-art AC motor drive consists of two key components: an electrical machine and power converter, as illustrated in Fig. 1.

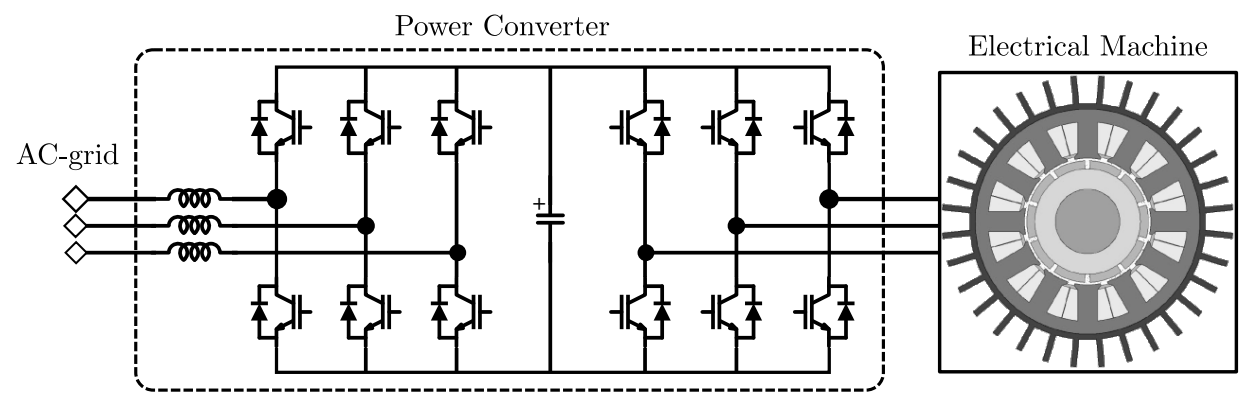

Fig. 1: Typical AC-motor drive topology consisting of an electrical machine and a power converter for AC grid connection.

In a motor drive, the power converter is usually more efficient compared to the electrical machine. However, its losses are a function of machine current and are not negligible, as they dictate the required 
converter cooling system volume and weight. For the electrical machine, a significant part of its iron loss is due to pulsed stator voltages with high harmonic content generated by power converters [3]. Further, converter DC-link voltage imposes a strict limit on the machine stator voltage and dictates the speed at which the machine needs to begin operating in field-weakening mode. For the best system weight and efficiency, these machine-converter interactions have to be taken into account and the design of these components have to be optimized together $[4,5]$.

Considering the complex multiphysics nature of a motor drive operation, a traditional design approach using generic sizing equations is labour intensive and rarely results in truly optimal designs. An alternative approach, also known as an optimization-based design [6], treats the design as an optimization problem and solves it using modern optimization algorithms on a computer. The advantage of the optimization-based approach is a significant reduction in engineering time and number of approximations/assumptions required.

Individual machine and converter component designs using this optimization-based approach are increasingly common and several examples can be found $[7,8,9]$. However, there are significantly fewer papers tackling motor drive designs, as a system rather than components, with this same approach. This is partly due to a lack of system-level motor drive models, that are computationally efficient and accurate enough to be employed at the core of the optimization [10].

Therefore, this paper presents a fast and accurate system-level motor drive model for an optimizationbased design, considering several aspects of the machine-converter interactions in details. Firstly, the general optimization-based design approach is introduced, followed by the development of a systemlevel motor drive model that is suitable to be used in this approach. The model is subsequently employed to analyse system-level effects for a prototype motor drive system, as a result of varying converter parameters and machine operating points. The analysis and findings are validated through experimental tests performed on a back-to-back motor drive test bench. A key feature of the model is that it is fully based on analytical equations and is non-iterative, making it very computationally efficient and, hence, suitable for use in an optimization-based design.

\section{Optimization-based design}

Motor drive designs, in general, involve analysis or modelling, to provide understanding of the realworld system, and optimization, to provide insight into designs that are optimal. The traditional design approach relies heavily on 'rule-of-thumbs' and sizing equations formulated by experienced engineers, combining elements of analysis and optimization. With this approach, significant simplifications and assumptions are usually in place, especially for complex problems with many input and output parameters. An optimization-based design approach instead directly employs the complex model in the design environment, keeping its basic input-output form for the design, as illustrated in Fig. 2.

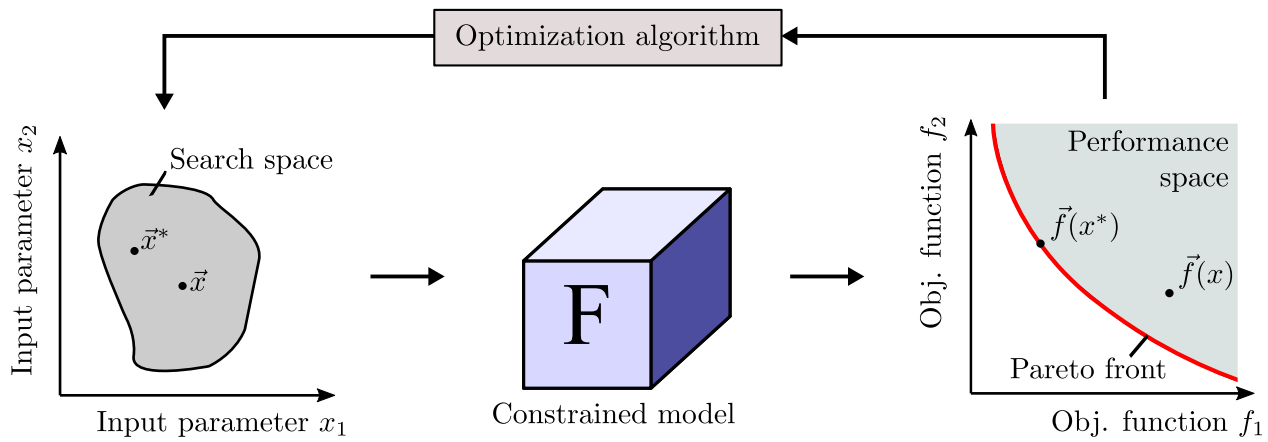

Fig. 2: Illustration of an optimization-based design where complex models map a set of design variables from search space onto the objective function space.

Using modern optimization algorithms like Genetic Algorithms (GA), input parameters to the model are varied and selected externally, over a number of generations, in search for a set of Pareto-optimal 
solutions. As the optimization process is handled by a computer, the required engineering time can be significantly reduced and simplified design equations with many assumptions are no longer necessary. This is especially beneficial for high dimensional, non-linear, highly constrained, and multi-objective design problems such as the motor drive design.

\section{System-level motor drive model}

This section describes the system-level motor drive model developed for optimization-based designs. Assuming a high power density motor drive application and an AC-power supply [11], the motor drive is considered to employ a surface-mounted Permanent Magnet Synchronous Machine (PMSM) for driving the mechanical load, and a two-level Voltage-Sourced Back-to-Back Converter (VSBBC) for AC-AC power conversion. In this work, component-level models for the PMSM and VSBBC are into a motor drive system-level model, as depicted in Fig. 3. Additional machine-converter interactions, such as machine harmonic losses are also included in this model.

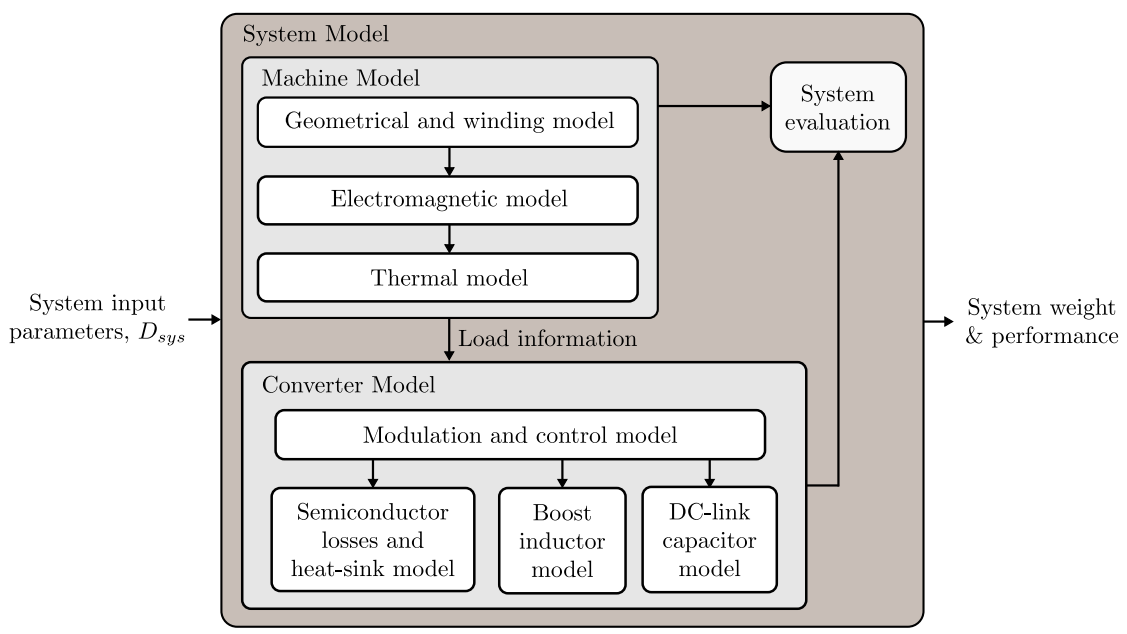

Fig. 3: Structure of the proposed motor drive system-level model.

The system-level model takes in a set of input parameters and outputs the combined weight and performances. Input parameters to the model include machine geometrical parameters, converter modulation parameters, values of passive components, etc. while its outputs include total weight, machine rated torque, machine flux densities, machine and converter's efficiency and steady-state temperatures, input current maximum peak-to-peak ripple, etc. The task of ensuring that certain performance constraints are met is handled externally by the optimization algorithm, by classifying solutions to be either feasible or infeasible solutions.

More details on the models are presented as follows:

\section{PMSM component model}

A simple structure is employed for the analytical machine model, consisting only of three stages as outlined in Fig. 3 [12]. Firstly, the full geometry and winding properties of the PMSM are calculated. Mathematical winding functions are employed to represent the optimal winding layouts for different slot-pole combinations.

In the second stage, the machine is evaluated in terms of its electromagnetic performance. The airgap/magnet region magnetic field due to the magnets and armature reaction are computed by solving the Laplacian/quasi-Poissonian field equations in the air-gap region. Slotting effects are accounted for using the conformal mapping technique. This analysis method is highly accurate, especially if saturation can be neglected. Based on the air-gap field distribution, the back-electromotive force, electromagnetic torque, magnetizing inductances, flux densities, and fundamental losses are determined. Machine leakage inductances are calculated from the slot and end-winding geometry assuming infinite permeability 
of the stator core. In the final stage, machine thermal behaviour is considered using a reduced-node Lumped Parameter Thermal Network (LPTN) to predict its maximum steady-state operating temperatures $[15,16]$.

\section{VSBBC component model}

A two-level VSBBC topology consists of two stages: Active Front End (AFE) rectifier stage and Voltage Source Inverter (VSI) stage, as depicted in Fig. 1. The power converter model consists only four parts, as outlined in Fig. 3 [17].

The modulation and control of the converter are firstly characterized as they significantly impact its operation and electrical behaviour. A fixed switching frequency modulation enables an exact calculation of the 'on' or 'off' timings for each switching device under a given modulation scheme. This subsequently allows average duty-cycle references, average and RMS squared currents of devices to be calculated over each fundamental period.

For control, a standard cascaded control scheme is adopted for the AFE and VSI. The VSI is operated under machine speed and output current control, while the AFE is run under DC-link voltage and input current control.

Semiconductor device properties are obtained from manufacturers and used for the semiconductor loss calculations. Based on device average and RMS squared currents, the total conduction loss is computed. On the other hand, total switching loss is determined from the device blocking voltage, instantaneous current and modulation patterns. To ensure that switching devices operate below a maximum junction temperature, a minimum cooling system volume is required, estimated based on a thermal resistance against volume relationship. In this work, a forced-air convection cooling system is assumed.

Finally, the main passive components, which are the DC-link capacitors and input boost inductors, are sized physically based on commercially available products [17]. For the boost inductors, distributed-gap cores with powdered iron-based alloys are considered due to their soft saturation characteristics. For the DC-link capacitors, polypropylene film capacitors are chosen because of their better temperature characteristics and current ripple tolerances.

\section{Machine harmonic loss model}

One important aspect of the machine-converter interaction modelled here also is machine harmonic loss due to harmonic content of the distorted pulsed converter voltage. An approach similar to the 'loss factor' method proposed in [13] is adopted, assuming dominant eddy-current loss over conductor loss and hysteresis loss at high harmonic frequencies. With a distorted stator winding current, eddy-current $I_{a c, h}$ induced in conductive parts of the machine is:

$$
I_{a c, h}=-\frac{\mathrm{d} \Psi_{h}}{\mathrm{~d} t} \cdot \frac{1}{R_{a c, h}} \quad \rightarrow \quad I_{a c, h} \propto-\frac{I_{h, r m s} f_{h}}{R_{a c, h}}
$$

where $\Psi_{h}$ is the harmonic flux component created by stator winding harmonic currents $I_{h, r m s}$ at frequency $f_{h}$.

Eddy-current losses $P_{a c, h}$, due to $I_{a c, h}$, are:

$$
P_{a c, h}=R_{a c, h} I_{a c, h}^{2} \propto \frac{I_{h, r m s}^{2} f_{h}^{2}}{R_{a c, h}}
$$

where $R_{a c, h}$ is the AC resistance of these conductive parts at harmonic frequency $f_{h}$. For a round conductor, $R_{a c, h}$ is proportional to $\sqrt{f_{h}}$ due to skin effect, as:

$$
R_{a c, h} \propto \frac{1}{S_{h}} \propto \frac{1}{d_{h}} \propto \sqrt{f_{h}}
$$

where $S_{h}$ is the cross-sectional area of conduction, and $d_{h}$ is the skin depth of the round conductor. For the 
complex geometry in which eddy-currents are induced, it is proposed to consider $R_{a c, h}$ to be proportional to $f_{h}^{x}$ instead, where $x$ is determined experimentally.

For different modulation methods, closed-form solutions for $I_{h, r m s}$ of a two-level voltage source converter are derived by integrating the average squared value of instantaneous current ripples over a fundamental cycle. According to Holmes et al. [14], $I_{h, r m s}$ can be calculated as:

$$
I_{h, r m s}^{2}=\left(\frac{V_{d c}}{L_{h} f_{h}}\right)^{2} \frac{1}{48} f(M)
$$

where function $f(M)$ is the harmonic distortion factor specific to different modulation methods, $M$ is the modulation index, $L_{h}$ is the machine inductance at $f_{h}$, and $V_{d c}$ is the DC-link voltage.

Substituting (3) and (4) into (2) gives

$$
P_{a c, h} \propto\left(\frac{V_{d c}^{2}}{L_{h}^{2} f_{h}}\right) \frac{1}{48} f(M)\left(\frac{f_{h}^{2}}{f_{h}^{x}}\right)
$$

Simplifying (5) and considering only the switching frequency components, the following harmonic loss model is proposed:

$$
P_{a c, h}=K_{a} V_{d c}^{2} f(M) f_{s w}^{K_{b}}+K_{c}
$$

where coefficients $K_{a}, K_{b}$, and $K_{c}$ are determined experimentally for a specific machine.

\section{System-level analysis and experimental verifications}

As a whole, the system model is simple and can take in many design variables. Various machineconverter interactions can be analysed with this model. In this paper, two specific aspects of the interactions are discussed:

1. Effects of converter modulation parameters on system loss, considering trade-off between converter switching loss and machine harmonic loss.

2. Effects of machine operating points on system loss, with different converter DC-link voltages and field-weakening enabled at higher machine speeds.

For each analysis, experimental tests are also performed to verify the findings and, thus, the accuracy of the model. Firstly, the setup, on which the experimental tests are performed, is discussed.

\section{Experimental setup}

The test bench consists of a pair of back-to-back motor-drive system connected at both their electrical and mechanical interfaces, forming a closed power loop transfer. This setup allows for loss measurements at a wide range of operating points. A photograph and basic schematic of the setup is shown in Fig. 4.

At the DC-link, a DC voltage supply regulates the voltage and supplies power to Conv $_{1}$, which drives $\mathrm{PMSM}_{1} . \mathrm{PMSM}_{1}$, in turn, mechanically drives $\mathrm{PMSM}_{2}$ which regenerates power through the Conv 2 back to the DC-link. Essentially, the DC voltage supply only supplies loss occurring in the loop, due to power conversions and transmission. Kinetiq PPA2500 precision power analysers are connected at each electrical interface and a torque transducer HBM T40b is placed at the mechanical connection of both machines to measure power loss across each individual components.

In this setup, the machines employed are two identical prototype 12-slot 10-pole surface mounted PMSMs while the semiconductor switching devices in the converters are Infineon FS50R12KT4 power modules. For the tests, a converter-machine pair can be considered as the drive system under test whilst the other as the loading drive system. The loading drive system is operated under speed control, regulating the shaft speed, while the drive system under test is operated under current control to set the torque.

With this setup, the individual loss distribution and efficiencies of each components can be measured at 


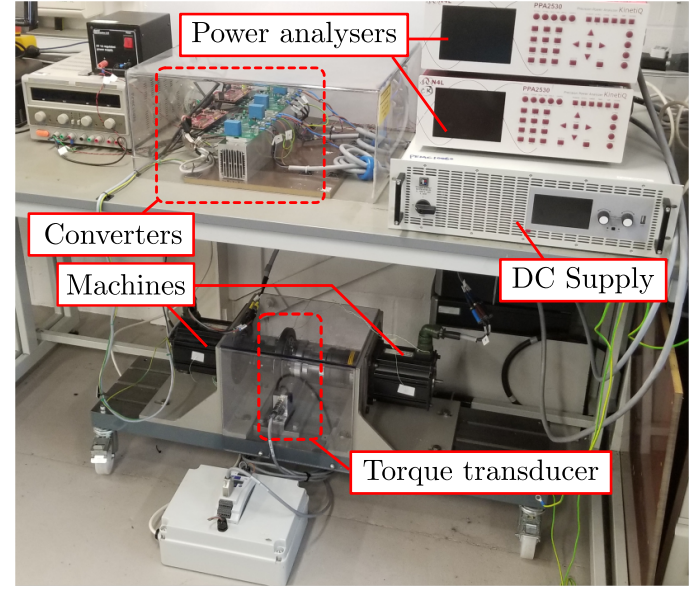

(a)

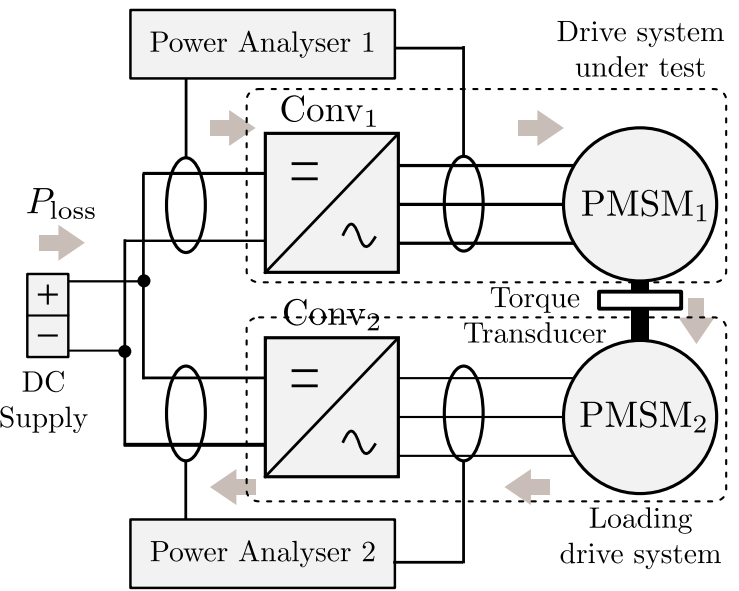

(b)

Fig. 4: (a) Photograph and (b) basic schematic diagram of the back-to-back motor drive system test bench (grey arrows represent direction of power flow).

various machine operating points and converter modulation parameters without an external load bank. Further information of the test setup are provided in Table I.

Table I: Specifications of back-to-back motor drive test setup.

\begin{tabular}{ccc}
\hline Parameter & Value & Unit \\
\hline Machine rated power & 1300 & $\mathrm{~W}$ \\
Machine rated speed & 1500 & $\mathrm{rpm}$ \\
Converter switching frequency & 4 to 20 & $\mathrm{kHz}$ \\
Converter DC-link voltage & 150 to 300 & $\mathrm{~V}$ \\
\hline
\end{tabular}

\section{Effects of converter modulation parameters on system loss}

Before the load tests, harmonic losses of the prototype machines are first measured for a range of DC-link voltages and switching frequencies to calculate the machine's harmonic loss parameters from (6). The measurements are taken at rated speed and quasi-no-load conditions, when only mechanical losses are occurring.

Firstly, the loading drive is mechanically disconnected from the drive system under test, and the drive is operated under speed control with a freely rotating shaft. The precision power analyser is then configured to automatically subtract the fundamental active power component from the total measured active power [4]. The measurement window is set to $800 \mathrm{~ms}$, and a linear averaging over 10 measurement values is performed. Assuming that harmonic power generates negligible mean torque, differences between total measured active power and fundamental active power component are considered to be the harmonic loss generated. As machine mechanical losses are typically low, the precision power analyser automatically switches to its higher accuracy current shunt for more accurate harmonic power loss measurements.

The measured losses are plotted on Fig. 5 as black dots. From these data points, a curve-fitting surface is constructed, based on the proposed harmonic iron loss model in (6) with a least-squares approximation method. For the prototype PMSM machines, the resulting curve-fitted harmonic loss parameters are $0.01943,-0.3168$, and -2.676 , for $K_{\mathrm{a}}, K_{\mathrm{b}}$, and $K_{\mathrm{c}}$ respectively, with an R-Square of 0.9973 .

With these harmonic loss parameters, system loss distributions, under rated power and speed conditions but different converter modulation parameters, are predicted by the system model to consider the tradeoff between converter switching loss and machine harmonic loss. Results from this analysis are shown in Fig. 6(a). 


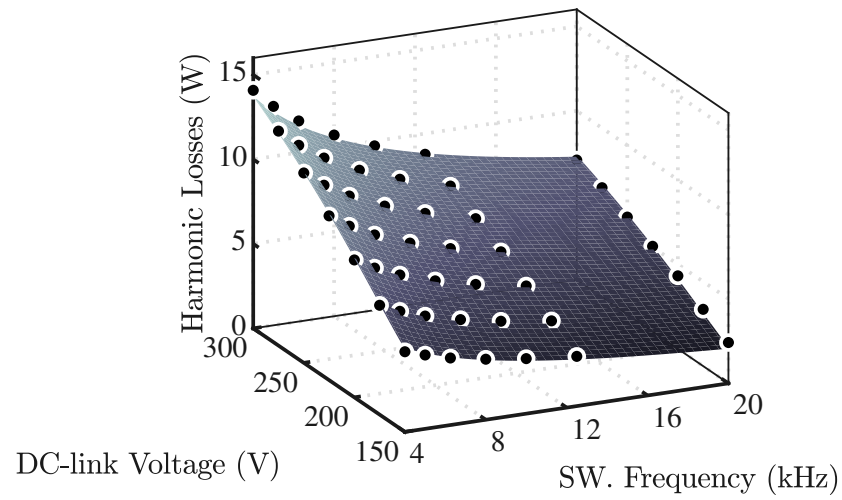

Fig. 5: Plot of harmonic loss measurements for prototype PM machine against switching frequency and DC-link voltage.

On one hand, least amount of system loss is produced when converter DC-link voltage is lowest as both converter switching loss and machine harmonic loss are positively correlated to the DC-link voltage. On the other hand, least amount of system loss is also produced when converter switching frequency is lowest, despite the negative correlation of machine harmonic loss with DC-link voltage. This is because converter switching loss at high load current is predicted to be significantly larger than machine harmonic loss.

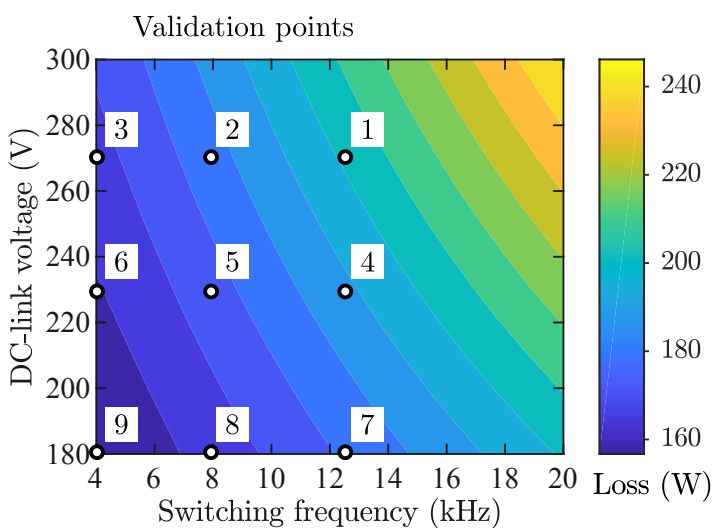

(a)

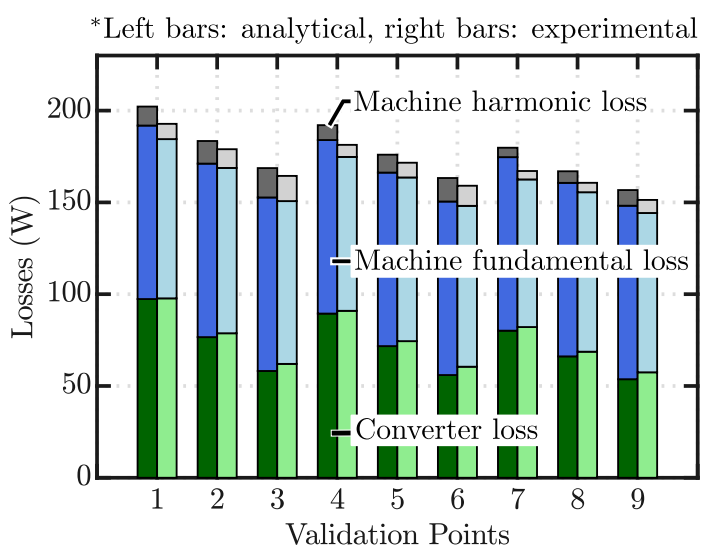

(b)

Fig. 6: (a) Plot of total losses against DC-link voltage and switching frequency calculated by the systemlevel model. The circled dots represent the selected experimental validation points. (b) Comparison of system-level model losses against measured losses at the validation points.

To verify this analysis experimentally, the loading drive is reconnected to the drive system under test, and the drive is now operated under rated power and speed conditions. Nine validation points, with different DC-link voltage and VSI switching frequency, are chosen, as indicated in Fig. 6(a), and the measured loss distributions at rated current and no-load conditions are compared against model predictions in Fig. 6(b).

A reasonable match was obtained between the model predictions and experimental measurements, validating the model's accuracy. As can be seen from Fig. 6(b), the increase in machine harmonic loss is not significant compared to the decrease in switching loss when a lower converter switching frequency is employed.

\section{Effects of machine operating points on system loss}

In most motor-drive applications, the system has to accommodate a wide range of operating points, as specified in their load profiles. These operating points are often defined in terms of torque and speed. 
Considering a simple traction motor drive torque-speed curve: constant rated torque is required up to a rated speed, from which point onwards only constant power is required, as illustrated in Fig. 7(a). To not exceed a given converter modulation index limit, field-weakening is often needed beyond a certain speed which is determined by the machine stator voltage and converter DC-link voltage.

For motor drive with a fixed PMSM design, the choice of DC-link voltage is a trade-off between system efficiency in the constant torque region and constant power region.

1. In the constant torque region, it is desirable to have as low a DC-link voltage value as possible, to reduce converter switching losses

2. In the constant power region, however, a low DC-link voltage can force the drive to enter into field-weakening mode at lower speeds. In field-weakening mode, due to the extra d-axis current component, machine winding and semiconductor losses are higher. Therefore, for higher efficiency in the constant power region, it is instead desirable to have a higher DC-link voltage and avoid field-weakening.

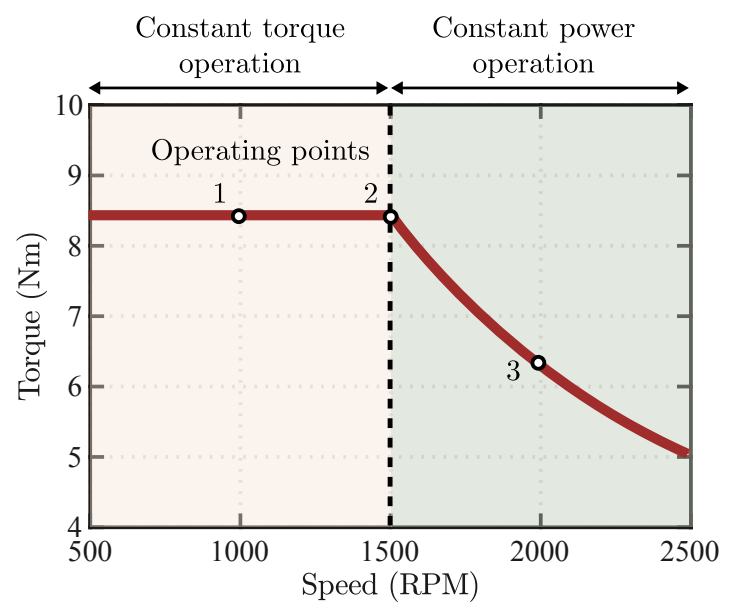

(a)

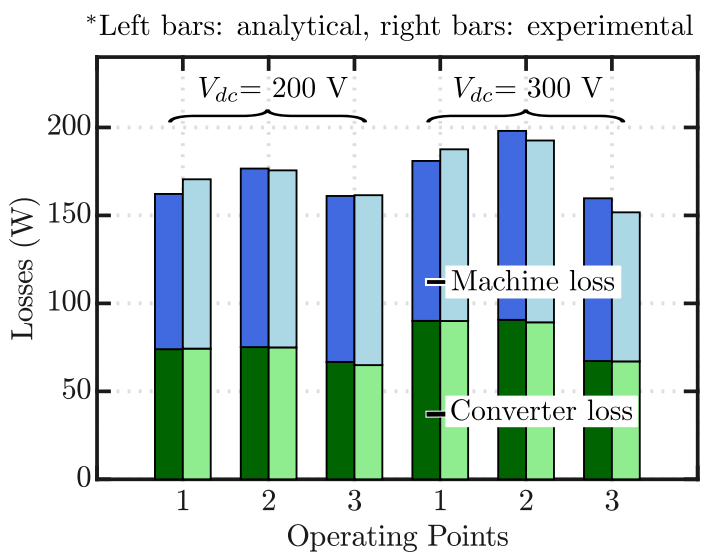

(b)

Fig. 7: (a) Torque-speed curve for a traction motor drive application. (b) Comparison of system model losses against measured losses for each validation point, with different DC-link voltages.

To illustrate this trade-off, three operating points on the torque-speed curve of Fig. 7(a) are chosen and their losses are evaluated using the system model for two different DC-link voltages (200 and $300 \mathrm{~V}$ ). Similarly, these operating points are realised on the experimental test bench and the losses are measured. Results of the predicted and measured losses are shown in Fig. 7(b), reporting similar findings.

With a lower DC-link voltage (200 V), system losses at operating points 1 and 2 (constant torque region) are observed to be lower compared to when a higher DC-link voltage $(300 \mathrm{~V})$ is employed. At operating point 3 (constant power region), however, the opposite is true, i.e. losses for a higher DC-link voltage $(300 \mathrm{~V})$ is lower compared to a lower DC-link voltage $(200 \mathrm{~V})$. As explained, this is due to the fieldweakening operation at higher speeds for the lower DC-link voltage system.

The above highlights the importance of selecting an appropriate converter DC-link voltage considering how it affects the average system losses across all the required operating points of its load profile. If the drive system is operated for long periods of time in the high speed region, a higher DC-link voltage will give a lower average system loss whilst the opposite is true.

The analysis thus far has assumed a fixed machine design, considering only effects of the converter DClink voltage on the drives operation and losses. It is desirable, alongside the DC-link voltage, to also optimise the machine design, in terms of its flux linkage (which relates to its voltage to speed ratio) and inductance (which dictates its flux-weakening capability), and further improve its efficiency. 


\section{Conclusion}

Design optimization of motor drive systems is challenging as it involves modelling the complex and multiphysics interaction between its main components. Further, the models have to be developed according to the design environment that it will be used within. This paper presented a system-level motor drive model that is computationally efficient and accurate, making it suitable for use in optimization-based designs. The model is kept to its basic input-output form and its accuracy has been validated through experimental tests for a particular machine and converter. Further verifications of the model will be performed on machines and converters with different power and speed ratings, and findings will be reported in future publications.

\section{References}

[1] M. Lukic, A. Hebala, P. Giangrande, C. Klumpner, S. Nuzzo, G. Chen, C. Gerada, C. Eastwick, and M. Galea State of the Art of Electric Taxiing Systems, in 2018 IEEE ESARS-ITEC, Nottingham UK, pp. 1-6, 2018.

[2] V. Madonna, P. Giangrande and M. Galea, Electrical Power Generation in Aircraft: Review, Challenges, and Opportunities, in IEEE Transactions on Transportation Electrification, vol. 4, no. 3, pp. 646-659, Sept. 2018.

[3] A. Al-Timimy, G. Vakil, M. Degano, P. Giangrande, C. Gerada, and M. Galea: Considerations on the Effects that Core Material Machining has on an Electrical Machines Performance, IEEE Transaction on Energy Conversion, vol. 33, no. 3, pp. 1154-1163, 2018.

[4] M. Schweizer, T. Friedli and J. W. Kolar, "Comparative Evaluation of Advanced Three-Phase Three-Level Inverter/Converter Topologies Against Two-Level Systems," in IEEE Transactions on Industrial Electronics, vol. 60, no. 12, pp. 5515-5527, Dec. 2013

[5] A. Balamurali, G. Feng, C. Lai, V. Loukanov and N. C. Kar, 'Investigation into variation of permanent magnet synchronous motor-drive losses for system level efficiency improvement," IECON 2017 - 43rd Annual Conference of the IEEE Industrial Electronics Society, Beijing, 2017, pp. 2014-2019.

[6] SD. Sudhoff, "Power magnetic devices: a multi-objective design approach" John Wiley \& Sons, 2014.

[7] M. D. Nardo, G. L. Calzo, M. Galea and C. Gerada, "Design Optimization of a High-Speed Synchronous Reluctance Machine," in IEEE Transactions on Industry Applications, vol. 54, no. 1, pp. 233-243, Jan.-Feb. 2018.

[8] S. G. Min, G. Bramerdorfer and B. Sarlioglu, "Analytical Modeling and Optimization for Electromagnetic Performances of Fractional-Slot PM Brushless Machines," in IEEE Transactions on Industrial Electronics, vol. 65, no. 5, pp. 4017-4027, May 2018.

[9] I. Laird, X. Yuan, J. Scoltock and A. J. Forsyth, "A Design Optimization Tool for Maximizing the Power Density of 3-Phase DCAC Converters Using Silicon Carbide (SiC) Devices," in IEEE Transactions on Power Electronics, vol. 33, no. 4, pp. 2913-2932, April 2018.

[10] D. arko, M. Kovai, S. Stipeti and D. Vuljaj, "Optimization of electric drives for traction applications," 2017 19th International Conference on Electrical Drives and Power Electronics (EDPE), Dubrovnik, 2017, pp. 15-32.

[11] P. Giangrande, A. Galassini, S. Papadopoulos, A. Al-Timimy, G. Lo Calzo, M. Degano, C. Gerada and M Galea, Considerations on the development of an electric drive for a secondary flight control electromechanical actuator, in press on IEEE Transactions on Industry Applications, pp. 11, 2019.

[12] Cheong, B., Giangrande, P., Zhang, XC., Galea, M., Zanchetta, P., and Wheeler, P., 'Fast and accurate multiphysics model for optimization-based design of fractional-slot PM machines," ICEMS 2019 - The 22nd International Conference on Electrical Machines and Systems, Harbin, 2019 (To be published)

[13] Bradley K., Cao W., Clare J., and Wheeler P., Predicting inverter-induced harmonic loss by improved harmonic injection IEEE Transactions on Power Electronics, vol. 23, no. 5, pp. 26192624, sep 2008.

[14] Holmes D. G. and Lipo T. A., "Pulse Width Modulation for Power Converters: Principles and Practice." John Wiley \& Sons Inc. 2003

[15] P. Giangrande, V. Madonna, S. Nuzzo, C. Spagnolo, C. Gerada, and M. Galea, ”Reduced Order Lumped Parameter Thermal Network for Dual Three-Phase Permanent Magnet Machines" in 2019 IEEE Workshop on Electrical Machines Design Control and Diagnosis, Athens, Greece, April 2019.

[16] V. Madonna, A. Walker, P. Giangrande, C. Gerada, G. Serra, and M. Galea, 'Improved thermal management and analysis for stator end-windings of electrical machines," IEEE Transactions on Industrial Electronics, pp. 1-1, 2018.

[17] B. Cheong, P. Giangrande, M. Galea, P. Zanchetta and P. Wheeler, 'Integrated motor drive design for weight optimization," 2017 IEEE Energy Conversion Congress and Exposition (ECCE), Cincinnati, OH, 2017, pp. 816-823. 